\title{
Antitumor effects of PLGA nanoparticles encapsulating the human PNAS-4 gene combined with cisplatin in ovarian cancer
}

\author{
XIAORONG QI*, XIANGRONG SONG*, PING LIU, TAO YI, SHUANGZHI LI, CHUAN XIE, \\ YU ZHENG, YU BAI, CHUNTANG SUN, YUQUAN WEI and XIA ZHAO
}

\begin{abstract}
Department of Gynecology and Obstetrics, Key Laboratory of Obstetric and Gynecologic and Pediatric Diseases and Birth Defects of Ministry of Education, West China Second Hospital, State Key Laboratory

of Biotherapy, Sichuan University, Chengdu 610041, P.R. China
\end{abstract}

Received March 12, 2011; Accepted May 5, 2011

DOI: $10.3892 /$ or.2011.1337

\begin{abstract}
Human PNAS-4 (hPNAS-4), as a pro-apoptotic gene, can inhibit tumor growth when overexpressed in some malignant cells. Poly (lactic-co-glycolic acid) (PLGA) was used as a gene transfer vector due to the advantage of sustained release, nontoxicity and biodegradability. In this study, we aimed to investigate the effect of PLGA nanoparticles encapsulating hPNAS-4 combined with cisplatin (DDP) on ovarian carcinoma. Expression of hPNAS-4 was determined by RT-PCR. Mice bearing intraperitoneal ovarian carcinomas were treated with PBS, pVAX-PLGA nanoparticles (P-P), pVAX-hPNAS4-PLGA nanoparticles (PhP-P), DDP and PhP-P plus DDP, respectively. Intraperitoneal tumors were weighed to assess the antitumor efficacy. The percentage of proliferative cells and apoptotic cells was evaluated by Ki-67 staining and terminal deoxynucleotidyl transferase-mediated dUTP nick end labeling. The anti-angiogenic effects were detected by CD31 staining and the alginate-encapsulate assay. Overexpression of hPNAS-4 was detected by RT-PCR in the PhP-P and PhP-P plus DDP groups. PhP-P exerted significant antitumor activity through induction of apoptosis, inhibition of cell proliferation and suppression of angiogenesis, compared with treatment with P-P or PBS alone. The combination of PhP-P with DDP showed enhanced antitumor activity compared with therapy of PhP-P or DDP alone. PLGA encapsulating hPNAS-4 combined with DDP may have promising applications in the therapy of ovarian cancer.
\end{abstract}

Correspondence to: Professor Xia Zhao, Department of Gynecology and Obstetrics, Key Laboratory of Obstetric and Gynecologic and Pediatric Diseases and Birth Defects of Ministry of Education, West China Second Hospital, State Key Laboratory of Biotherapy, Sichuan University, No. 20, Section 3, South People's Road, Chengdu 610041, P.R. China

E-mail: xia-zhao@126.com

*Contributed equally

Abbreviations: PLGA, poly (lactic-co-glycolic acid); PBS, phosphatebalanced solution; DDP, cisplatin; PLL, polylysine; RT-PCR, reverse transcription-polymerase chain reaction

Key words: human PNAS-4, poly (lactic-co-glycolic acid), nanoparticles, ovarian cancer

\section{Introduction}

PNAS-4 is identified as a novel pro-apoptotic gene, which can inhibit tumor cell growth through apoptosis. It is activated in the early stage of DNA damage, and consequently exerts the function of inducing apoptosis. Previous studies have shown that PNAS-4 significantly inhibits tumor growth of CT26 colon carcinoma and LL2 lung carcinoma in murine models (1). PNAS-4 has been proven to be a key regulator of cell migration during gastrulation in zebrafish model (2). X. laevis PNAS-4 (xPNAS-4) has been found to be present in early embryo development (3). PNAS-4 was widely expressed in various tissues especially in skeletal muscle (4). The sequence identity between human PNAS-4 (hPNAS-4) protein and mouse, Xenopus and zebrafish PNAS-4 is 96, 87 and $81 \%$ respectively (5). Overexpression of hPNAS-4 has been proven to possess the biological activity of inhibiting cell proliferation via inducing cell apoptosis in some tumor cells, such as osteosarcoma, lung carcinoma and ovarian cancer (5-7). Filippov et al have reported that overexpression of hPNAS-4 in the human osteosarcoma cell line-U2O increased cell death via apoptosis (6). The apoptotic mechanisms of hPNAS-4 include activation of a mitochondrial pathway and arrest of the cell cycle in the $S$ phase (5).

Ovarian cancer is the leading cause of gynecological cancer mortality. There are an estimated 230,000 new cases of ovarian cancer, resulting in over 140,000 deaths every year (8). Cisplatin (DDP), one of the most important chemotherapy drugs, has been widely used to treat ovarian cancer. However, DDP action on malignant cells is elicited at high DDP doses. The severe toxicity and side-effects owing to the high dose often cause patient intolerance. As a result, a strategy using dual agents rather than a single agent may become a more useful alternative with the purpose of higher antitumor efficiency and lower toxicity. The antitumor effect of DDP derives from its capability to form bifunctional DNA crosslinks, failure to repair damaged DNA, interference with DNA replication, cell cycle arrest, and ultimately apoptosis (10-13). Apoptosis induced by hPNAS-4 also involves DNA damage $(8,14)$. Therefore, in this study, we aimed to utilize the combination therapy of hPNAS-4 with DDP to treat ovarian cancer.

Therapeutic efficiency of functional genes mainly depends on effective gene delivery systems $(14,15)$. At present, non-viral 
gene delivery systems and transfer techniques including the cationic liposome, the polymer-based system, the biolistic system, ultrasound, electroporation and hydroporation, have been widely studied. Nevertheless, the clinical use of these techniques is restricted due to cytotoxicity, low stability, trauma in operation and so on. In our previous study, it has been demonstrated that hPNAS-4 delivered by liposome complex has an effect on inhibiting tumor growth. However, the dose of plasmid required to achieve therapeutic effect was very high $(1,16)$, which restricts its future clinical use. The poly (lactic-co-glycolic acid) (PLGA) nanoparticles have the characteristics of nontoxicity, controlled and sustained release of embedded drug, rapid evasion of the endo-lysosomal pathway, selective deposition in target tissues, biocompatibility and biodegradability (17-20). Furthermore, PLGA nanoparticles have been approved by the US Food and Drug Administration (FDA) for the use of drug delivery. Accordingly, we used PLGA nanoparticles encapsulating the hPNAS-4 gene combined with DDP to investigate the antitumor efficiency of combination therapy on ovarian cancer.

In this study, the hPNAS- 4 exerted significant effectiveness in inhibiting tumor growth and angiogenesis. The antitumor activity of hPNAS-4 was enhanced when combined with DDP. Additionally, PLGA show efficient transfection without obvious toxicity.

\section{Materials and methods}

Cell culture and transfection. The human epithelial serous cystadenocarcinoma cell line SKOV3 was purchased from American Type Culture Collection (ATCC, Rockville, MD) and cultured in RPMI-1640 culture medium with $10 \%$ fetal calf serum, $2.0 \mathrm{mM}$ L-glutamine and $0.1 \mathrm{mg} / \mathrm{ml}$ amikacin. Cells were incubated at $37^{\circ} \mathrm{C}$ in a humidified atmosphere of $5 \% \mathrm{CO}_{2}$. SKOV3 cells $\left(2 \times 10^{5} /\right.$ well) were seeded in 6-well dishes and incubated to $80 \%$ confluence. pVAX-PLGA nanoparticles (P-P) and pVAX-hPNAS-4-PLGA nanoparticles (PhP-P) (containing $4 \mu \mathrm{g}$ plasmid) were mixed in the medium. DDP was prepared at a concentration of $5.0 \mu \mathrm{g} / \mathrm{ml}$. After incubation for $6 \mathrm{~h}$, the medium was replaced. Untreated cells were used as controls. After transfection, SKOV3 cells were collected for RT-PCR analysis.

Plasmid construction and purification. The pVAX vector (Invitrogen, Carlsbad, CA) encoding the hPNAS-4 (pVAXhPNAS-4) was a gift from Dr Ping Chen. The pVAX vector without hPNAS-4 gene was used as an empty control. Largescale plasmid DNA was prepared using the Endofree Plasmid Giga kit (Qiagen, Chatsworth, CA). The purified plasmids were dissolved in Tris-EDTA buffer and stored at $-20^{\circ} \mathrm{C}$ for further use.

PLGA nanoparticles formulation. The PLGA (75:25; MW, 50000) nanoparticles with cDNA were prepared by the following modification of a previously described water-in-oilin-water $(\mathrm{w} / \mathrm{o} / \mathrm{w})$ double emulsion solvent evaporation process (21). Briefly, cDNA was mixed with polylysine (PLL, MW, 25000, Sigma) - 5\% dextrose in water (5\% GS) for $30 \mathrm{~min}$. The cDNA was concentrated by electrostatic binding. PLGA dissolved in chloroform-acetone solution was dropped into the
cDNA-PLL mixture. The primary emulsion was formatted after the mixture was sonicated for $30 \mathrm{sec}$. Dispersed in 1\% $(\mathrm{w} / \mathrm{v})$ polyvinyl alcohol-Tris-EDTA buffer (PVA-TE), the primary emulsions were treated with sonication until the organic solvents were removed. The obtained nanoparticles were resuspended in deionized water and were stored at $4^{\circ} \mathrm{C}$ for further analyses. The PLGA nanoparticles prepared with pVAX were named P-P. The PLGA nanoparticles loaded with pVAX-hPNAS-4 were named PhP-P.

PLGA nanoparticles characterization. The particle size and polydispersity index (PDI) were determined by the Spectrex Laser Particle Counter. The process was repeated three times. PLGA nanoparticles loaded with cDNA were centrifuged $(15000 \mathrm{rpm}, 30 \mathrm{~min})$. The supernatant $(200 \mu \mathrm{l})$ was mixed into $3.8 \mathrm{ml}$ of the $0.15 \mu \mathrm{g} / \mathrm{ml}$ Hoechst 33258. The fluorescence intensity was determined at an excitation wavelength of $350 \mathrm{~nm}$ and an emission wavelength of $455 \mathrm{~nm}$. The plasmids in the supernatant which were not encapsulated in nanoparticles were quantified by a one point external standard method. The encapsulation efficiency (EE) of PhP-P was determined using the following formula: the ratio of the actual amount of encapsulated cDNA over the total amount of cDNA used for the preparation of the nanoparticles. The drug load (DL) was used to describe the actual amount of encapsulated cDNA over the amount of carrier materials. The surface of the PLGA nanoparticles was determined by the S-4800 Hitachi scanning electron micrograph (SEM) at x40,000 magnification. The average diameter of the PLGA nanoparticles was measured with photon correlation spectroscopy (PCS) and the Zetasizer Nano ZS90 potential analyzer (Malvern Instruments Ltd., Malvern, UK).

Detection of hPNAS-4 expression. RT-PCR was carried out to detect the expression of hPNAS-4 mRNA in vitro and in vivo. The upstream primer and downstream primer for hPNAS-4 (GenBank accession no. CAH70880) were: 5'-ccc agg aaa tgc ttc tgaac-3', and 5'-tga tcc agc gag gaa tct ct-3', respectively. The total-RNA was extracted by TRIzol reagent (Invitrogen) according to the manufacturer's instruction. RT-PCR was performed as follows: incubation at $52.7^{\circ} \mathrm{C}$ for $40 \mathrm{~min}$ and denaturation at $95^{\circ} \mathrm{C}$ for $3 \mathrm{~min}$; DNA amplification for 30 cycles $\left(95^{\circ} \mathrm{C}\right.$ for $30 \mathrm{sec}, 52.7^{\circ} \mathrm{C}$ for $30 \mathrm{sec}$ and $72^{\circ} \mathrm{C}$ for $30 \mathrm{sec} /$ cycle); a terminal elongation step at $72^{\circ} \mathrm{C}$ for $10 \mathrm{~min}$. The primers were used for RT-PCR amplification of hPNAS-4 (600 bp). GAPDH was used as an internal control (464 bp). The RT-PCR products were analyzed with $1.0 \%$ agarose gel electrophoresis.

Tumor model and treatment. Six to eight week-old female athymic nude mice were purchased from the Sichuan University Animal Center. SKOV3 cell line were originally injected s.c. to develop primary tumors; then the tumors were harvested for i.p. inoculation (22). Seven days after inoculation, therapy was initiated through intraperitoneal injection. The mice were randomly assigned into five groups to receive the following treatment (5 mice/group): a) PBS as control; b) P-P containing $5 \mu \mathrm{g}$ pVAX plasmid every 2 day for 12 times; c) PhP-P containing $5 \mu \mathrm{g}$ pVAX-hPNAS-4 for 12 times; d) DDP (Qinu Pharmacy Corporation, China) $3 \mathrm{mg} / \mathrm{kg}$ weekly for 3 times; e) PhP-P 

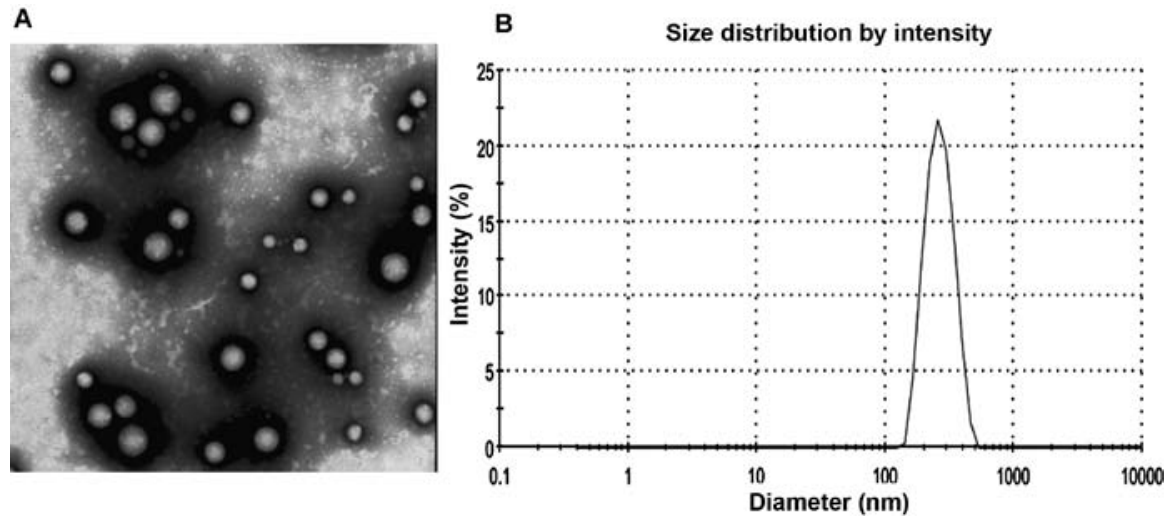

Figure 1. Characterization of PLGA nanoparticles. (A) Nanoparticles exhibited a uniform spherical shape with steadily dispersed suspension. The surface integrity of the PLGA nanoparticles and the absence of observable defects were observed under SEM (x40,000). (B) Representative DLS data for PhP-P.

plus DDP (PhP-P+DDP) containing treatment of PhP-P for 12 times and DDP for 3 times. Three days after the last treatment, mice were sacrificed. Tissues were fixed in $10 \%$ neutral buffered formalin and embedded in paraffin. All procedures of the animal experiments were approved by the Institute's Animal Care and Use Committee.

Immunohistochemical analysis. The tumor sections were deparaffinized and immersed in $10 \mathrm{mM}$ citrate buffer. Heatinduced antigen retrieval was accomplished in a pressure cooker. Endogenous peroxidase activity was quenched with $3 \%$ hydrogen peroxide. To reduce non-specific staining, the sections were incubated with $10 \%$ normal goat or rabbit serum. After overnight treatment with anti-mouse monoclonal CD31 antibody (Santa Cruz Biotechnology, Santa Cruz, CA) and anti-human monoclonal Ki-67 antibody (Thermo Fisher Scientific, Fremont, CA), sections were incubated with biotinylated secondary antibody and streptavidin-biotin-peroxidase complex (SABC), sequentially. The immunoreaction was visualized using the diaminobenzidine (DAB) solution as the chromogen, and then the cellular nuclei were counterstained with Mayer's hematoxylin.

The highest vascular density areas were identified at x 100 magnification. Five areas of highest microvessel density (MVD) were selected for counting at high-power field (hpf) (x400) (23). Cells with definite nuclear positive reactivity stained with $\mathrm{Ki}-67$ were counted in 10 random fields at hpf. The Ki-67 index depicting the positive cells among the total neoplastic cells was determined (24).

Apoptosis assay. Apoptosis in vivo was observed by terminal deoxynucleotidyl transferase-mediated dUTP nick end labeling (TUNEL) staining. The Dead End Fluorometric TUNEL System (Roche, Mannheim, Germany) was carried out following the manufacturer's instructions. Cell nuclei with apoptotic morphology, stained with green fluorescence were defined as TUNEL-positive. The number of TUNEL-positive nuclei was counted in 10 random fields at hpf $(x 400)$. The percentage of apoptosis was used to describe the number of apoptotic cells over the total number of cells (25).

Calcium alginate embedding. Alginate (Sigma) was autoclaved in $0.9 \% \mathrm{NaCl}$ solution at a concentration of $1.5 \%$ (w/v). SKOV3 cells were suspended in alginate solution. The alginate solution containing SKOV3 cells were released into a solution of calcium chloride $(250 \mathrm{mM})$. Microencapsulations containing SKOV3 cells $\left(2 \times 10^{5}\right.$ cells/bead $)$ were generated. Then, the microencapsulations were embedded at the back of mice ( 4 beads/mice) under anesthesia. The mice were treated with PBS, P-P, PhP-P, DDP or PhP-P plus DDP, respectively. In the end of the experiment, blood vessels covering calcium alginate beads were imaged under a microscope $(\mathrm{x} 4)$. Before the mice were sacrificed, $100 \mathrm{mg} / \mathrm{kg}$ FITC-dextran (Sigma) was injected through the tail vein. Microencapsulations marked with FITC-dextran were abraded and centrifuged in $0.9 \% \mathrm{NaCl}$ solution. The supernatants were evaluated with an enzyme-linked fluorescent immunoassay. The standard curve was determined with a series of FITC-dextran concentrations. The uptake of FITC-dextran was measured against the stadard curve.

Toxicology analysis. The relevant indices such as weight loss, ruffling of fur, behavior and feeding were evaluated continuously. The weight of mice was measured every three days. When the mice were sacrificed, discernible anomaly signs and inflammation in the abdominal cavity were assessed. Tissues and organs (including lung, liver, kidney, heart, pancreas and spleen) were fixed, embedded and sectioned. The sections were stained with hematoxylin and eosin (H\&E).

Statistical analysis. Data were assayed by analysis of variance (ANOVA) and the unpaired Student's t-test. All results were expressed as the means \pm standard error (SE). The statistical analyses were performed using the SPSS software (version 12.0). A P-value $<0.05$ was defined as statistically significant.

\section{Results}

Physical properties of nanoparticles. SEM images showed that PhP-P were uniform spheres. Additionally, the nanoparticles were free of observable defects (Fig. 1A). The average diameter and polydispersity index (PDI) of PhP-P were determined by PCS. The average diameter was $236.9 \pm 5.28 \mathrm{~nm}$ and the PDI was $0.16 \pm 0.01$. These data displayed a narrow size distribution, which was in agreement with the result of SEM. pVAX-hPNAS-4 was encapsulated into PLGA nanoparticles 


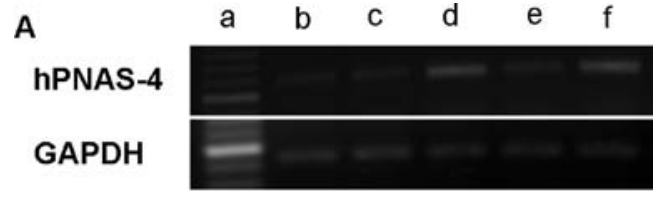

B

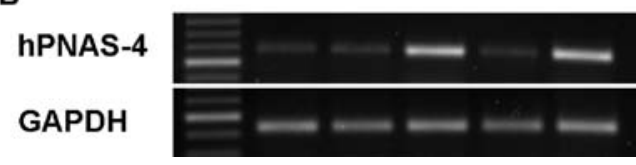

Figure 2. Expression of hPNAS-4 in vitro and in vivo. (A) RT-PCR analysis of the expression of hPNAS-4 in vitro. Overexpression of hPNAS-4 in SKOV3 cells transfected with PhP-P was detected by RT-PCR (600 bp). (B) RT-PCR analysis of hPNAS-4 expression in vivo. Overexpression of hPNAS-4 in tumor tissues treated with PhP-P was confirmed by RT-PCR. The panels of electrophoretic images present the following: DNA ladder (lane a), PBS (lane b), P-P (lane c), PhP-P (lane d), DDP (lane e) and PhP-P plus DDP (lane f), respectively. GAPDH was used for the internal standard (464 bp).

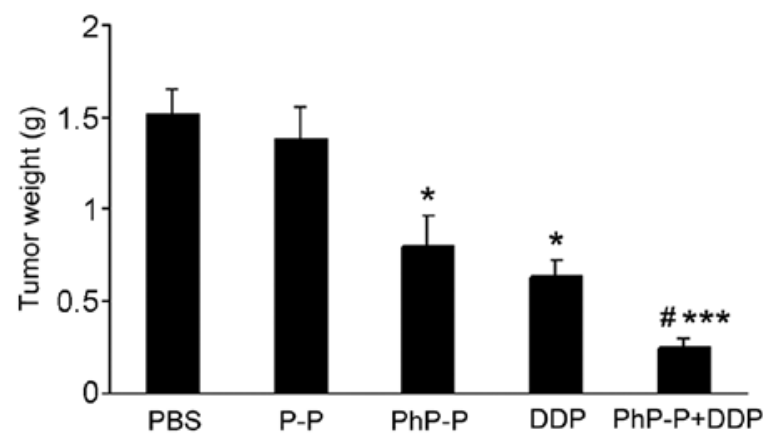

Figure 3. Tumor weights in the xenograft model of ovarian carcinoma in nude mice. The results indicate that PhP-P exhibited a similar effect of suppressing tumor growth with DDP ( $\mathrm{P}>0.05)$. The growth of ovarian cancer in the $\mathrm{PhP}-\mathrm{P}$ plus DDP group is significantly blocked ( $\mathrm{P}<0.05$ vs. any other group). However, the treatment of PBS and P-P did not exert the effect of inhibiting tumor growth. Results are expressed as the mean tumor weight \pm SE. Results were expressed as mean tumor weight \pm SE. "Indicates data points for which the PhP-P single-treatment group was significantly different from the combination therapy group $\left({ }^{\#} \mathrm{P}<0.05 ;{ }^{\# \#} \mathrm{P}<0.01\right)$. "Indicates significant differences compared with the PBS group $\left({ }^{*} \mathrm{P}<0.05 ;{ }^{* *} \mathrm{P}<0.01 ;{ }^{* * *} \mathrm{P}<0.001\right)$.

with a high EE of 91.24\%. Dynamic light scattering (DLS) further confirmed the homogeneity of the particles (Fig. 1B).

Expression of hPNAS-4 in vitro and in vivo. RT-PCR analysis was employed to verify whether transfection of PhP-P resulted in an evidently improved expression level of hPNAS-4 in ovarian cancer cells. Overexpression of hPNAS-4 was detected in SKOV3 cells transfected with PhP-P (600 bp) (Fig. 2A). The integrity and quantity of cDNA was confirmed by amplification of GAPDH (464 bp). Overexpression of hPNAS-4 in vivo was also conformed by RT-PCR after administration of PhP-P (Fig. 2B). The results show that overexpression of exogenous hPNAS-4 was detected in tumor tissues treated with PhP-P or PhP-P plus DDP. The results suggest that foreign hPNAS-4 gene loaded in PLGA nanoparticles could be expressed in ovarian cancer.

Tumor suppressor function of the combination of PhP-P with DDP. The group of animals treated with PhP-P alone exhibited a significant inhibition of tumor growth (mean tumor weight, 0.8 \pm 0.166 g) (Fig. 3). The tumor growth in the PhP-P group was significantly lower compared to those treated with P-P and PBS $(\mathrm{P}<0.05)$. This result was similar to what we observed in the group treated with DDP (mean tumor weight, $0.634 \pm 0.091 \mathrm{~g})(\mathrm{P}>0.05)$. The PhP-P + DDP-treated group showed an enhanced effect on tumor suppression (mean tumor weight, $0.248 \pm 0.048 \mathrm{~g}, \mathrm{P}<0.05)$. None of the nude mice in the three therapy groups developed blood ascites. The tumor weights of the P-P or PBS-treated group were obviously increased (mean tumor weight, $1.524 \pm 0.125,1.386 \pm 0.175 \mathrm{~g}$, respectively). Furthermore, macroscopic tumors and blood ascites were found in the two groups.

Inhibition of tumor cell proliferation in vivo. The proportion of proliferative tumor cells was analyzed by the percentage of Ki-67 positive cells (Fig. 4). Nuclear staining in tumor tissues of PhP-P or DDP single therapy groups showed less less staining for Ki-67 compared with the P-P and PBS groups $(\mathrm{P}<0.01)$. The mean percentage of $\mathrm{Ki}-67$ positive cells in the PhP-P group was not significantly different from that of DDP group $(\mathrm{P}>0.05)$. The percentage of Ki-67 positive cells in tumor tissues treated with PhP-P plus DDP was obviously reduced compared with the controls $(\mathrm{P}<0.01)$. The empty vector and PBS group exerted significantly tumor cell proliferation in vivo. A quantitative comparison of cell proliferation was shown in Fig. 4A.

Induction of apoptosis in vivo. The PhP-P and DDP monotherapy led to a significant increase apoptotic cells compared with the empty vector therapy $(\mathrm{P}<0.01)$. There was no significant difference in the $\%$ of apoptotic cells between the PhP-P and DDP group $(\mathrm{P}>0.05)$. The tumor tissues from the group treated with PhP-P plus DDP showed increased \% of apoptotic cells compared with the PhP-P or DDP groups $(\mathrm{P}<0.01)$. However, the empty vector and PBS group did not exhibit a cytopathic effect. A quantitative comparison of apoptosis is shown in Fig. 4B.

Inhibition of angiogenesis. Angiogenesis in tumor tissues were estimated by MVD assay and the quantification of the FITC-dextran uptake (Fig. 5). MVD was examined by CD31 staining. A significant reduction of MVD in PhP-P or DDP treated group was observed compared with that in the P-P or PBS treated group $(\mathrm{P}<0.05)$ (Fig. 5A). Combination therapy resulted in lower MVD compared with PBS group $(\mathrm{P}=0.001)$. However, in the P-P or PBS group, the increased microvessels were easily observed. Calcium alginate beads were performed to evaluated angiogenesis. There were few blood vessels in either the PhP-P or DDP monotherapy groups. Blood vessels were very rarely observed in the PhP-P plus DDP group. However, twisted and rich blood vessels around calcium alginate were found in the P-P and PBS groups. Compared with the empty vector group, decreased uptake of FITC-dextran was observed in the PhP-P and DDP monotherapy groups $(\mathrm{P}<0.01)$ (Fig. 5B). There was no significant difference between the PhP-P and the DDP groups $(\mathrm{P}>0.05)$. The concentration of FITC-dextran treated with PhP-P plus DDP was greatly reduced compared with monotherapy groups ( $\mathrm{P}<0.01$ vs. PhP-P group). In contrast, in empty plasmid or the 

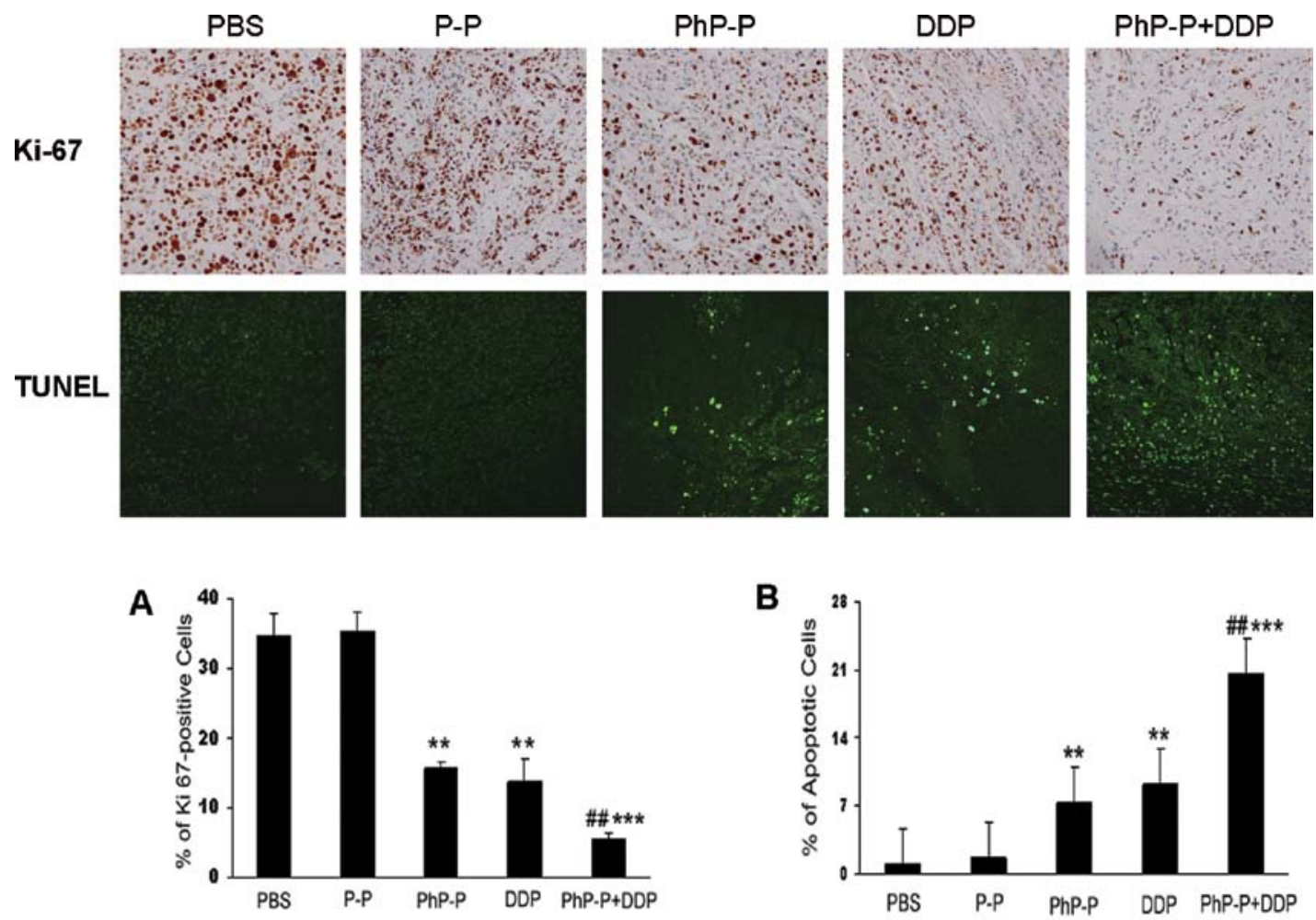

Figure 4. Effects of the treatments on SKOV3 cells assessed by Ki-67 and TUNEL immunostaining (x400). (A) Obvious suppression in cell proliferation was detected in the PhP-P group, which was similar to the DDP group $(\mathrm{P}>0.05)$. The tumor tissues of PhP-P plus DDP group revealed scarce Ki-67 nuclear staining. Ki-67 staining was found in the P-P group and PBS control group apparently. (B) Apoptotic cells were detected in tumors treated with PhP-P or DDP. Apoptotic cells were predominantly observed in the PhP-P + DDP group. Apoptotic cells were not apparent in the P-P or PBS groups.
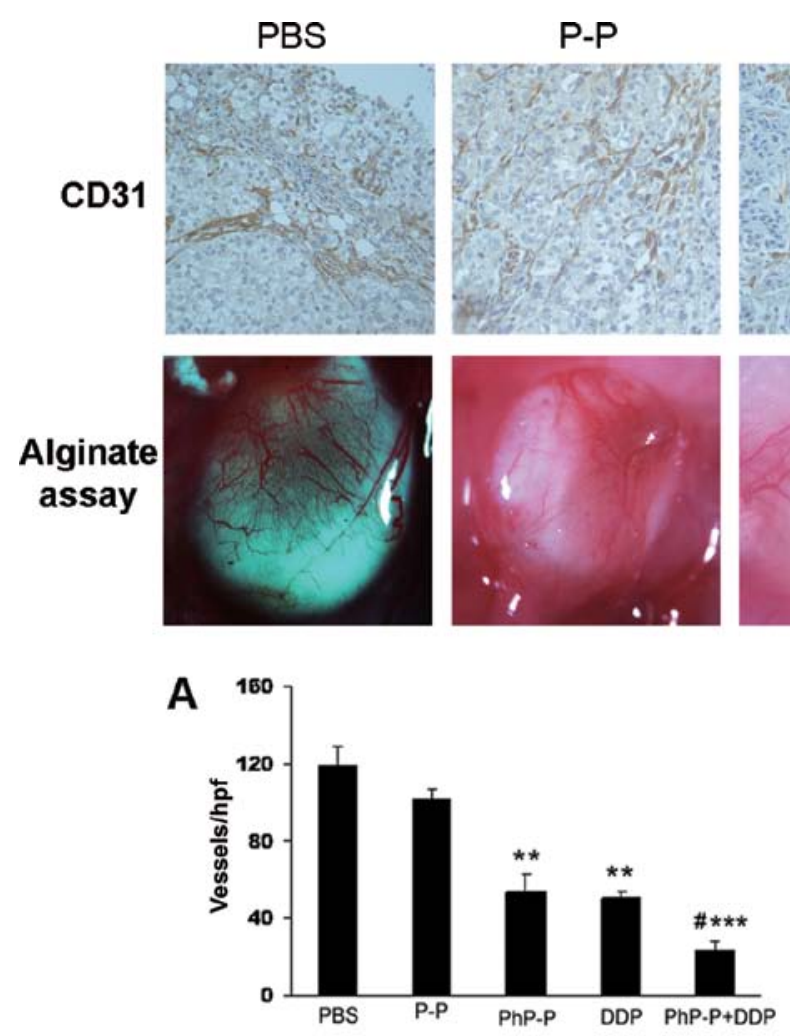
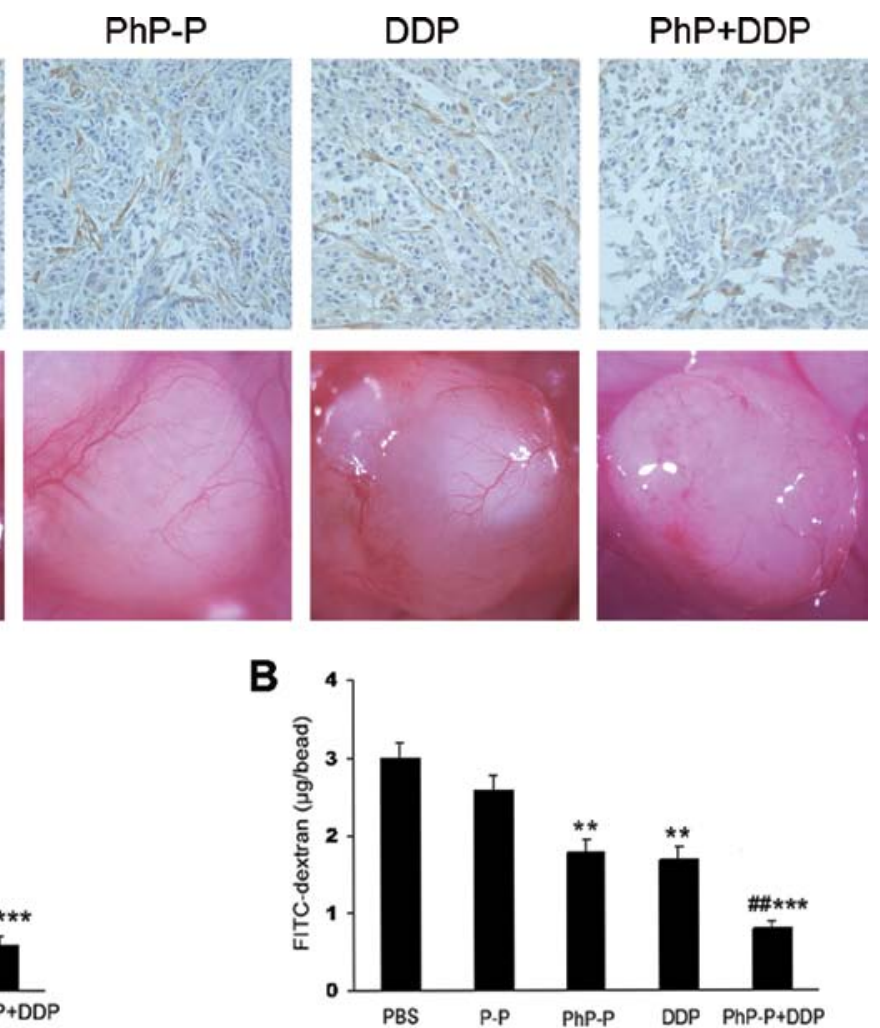

Figure 5. Effect on tumor angiogenesis was determined by CD31 immunostaining and calcium alginate embedding analysis in vivo. (A) Anti-CD31 staining revealed that there were sparse blood vessels in the tumor tissues of the PhP-P and DDP single therapy and very few blood vessels in the PhP-P + DDP-treated group; nevertheless, rich nascent capillary vessel were observed in the tumor tissues of the empty vector group and the PBS group. (B) The suppression of blood vessels occurred in the PhP-P and DDP singly treated groups. Statistically significant differences in the FITC-dextran uptake from mice in the PhP-P or DDP group vs. the PBS group were noted; There was a significant difference between the PhP-P + DDP and the PhP-P or DDP single treatment groups. 


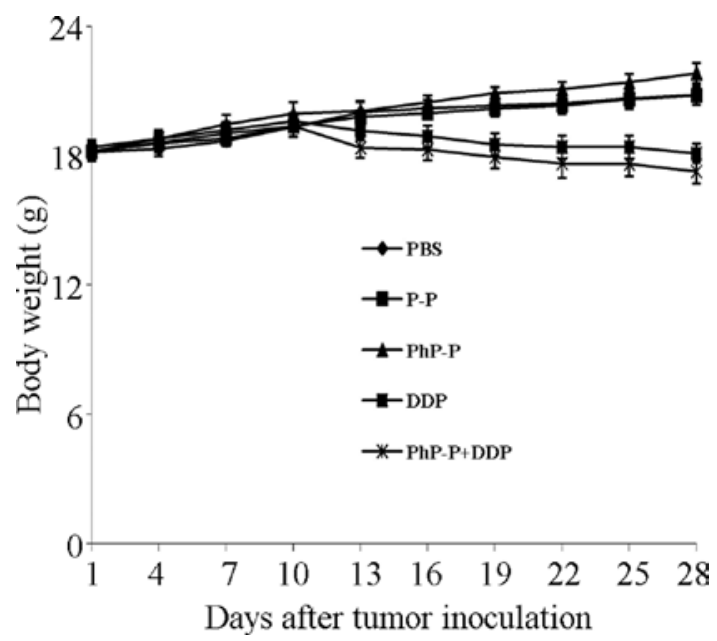

Figure 6. Mouse body weights. No significant differences in the weights of mice were found among the five groups $(\mathrm{P}>0.05)$. Weight loss occurred in the DDP and PhP-P + DDP-treated animals and no significant differences were found between the two groups.

PBS-treated group, an obviously increased uptake of FITCdextran was observed in comparison to the monotherapy group $(\mathrm{P}<0.01)$.

Safety. There was a slight decrease in the weights of mice observed in the DDP as well as the PhP-P plus DDP-treated animals and no significant differences were found between the two groups. However, no significant differences in the weight of mice were found among the five groups $(\mathrm{P}>0.05)$ (Fig. 6). Inflammation, incrassate or hyperemic peritoneum were also not been seen. Additionally, ruffling of the fur, abnormal behavior and feeding were not detected. Toxic reaction was also confirmed by morphometric analyses. The results of H\&E histological staining indicated that there were no significant pathological alterations in various organs (including lung, liver, kidney, heart, pancreas and spleen).

\section{Discussion}

hPNAS-4 could inhibit cell proliferation via apoptosis when overexpressed in several tumors. Filippov et al have reported that expression of hPNAS-4 was up-regulated in the human osteosarcoma cell line $\mathrm{U} 2 \mathrm{O}$ using the genotoxic agent mitomycin C (5). This finding indicated that hPNAS-4 may induce an apoptotic response due to DNA damage (6). Yan et al have observed a similar result in human lung adenocarcinoma (5). In their study, overexpression of hPNAS-4 in A549 cells resulted in down-regulation of Bcl-2 and up-regulation of Bax, and contributed to cytochrome $\mathrm{c}$ release from the mitochondria into the cytoplasm. This release led to caspase-3 cleavage downstream of cytochrome c. cleavage of caspase-3 resulted in the activation of endonuclease, consequently, leading to DNA fragmentation. In addition, overexpression of hPNAS-4 resulted in cell cycle arrest in the $S$ phase through regulating p21, E2F1, and proliferating cell nuclear antigen (5). Thus, overexpression of hPNAS-4 may relate to the suppression of DNA replication. The antitumor effect of DDP was involved in the formation of lethal intrastrand DNA crosslinks, interference with DNA replication, cell cycle arrest, and provocation of apoptosis (9-12). DNA damage inuduction might be the common antitumor mechanism for hPNAS-4 and DDP.

In this study, hPNAS-4 inhibited tumor cell proliferation and induced apoptosis. The antitumor effect in hPNAS-4 plus DDP was significantly enhanced relative to other agents, which was probably attributed to the common DNA damage-induced apoptotic pathway $(5,6,10)$. In our previous report, using other genotoxic agents, such as gemcitabine and honokiol plus hPNAS-4 resulted in a similarly enhanced effect $(1,7)$. Mouse PNAS-4 (mPNAS-4) combined with DDP in gene therapy has also been reported to enhance the inhibiting effect via apoptosis (26). These results are in agreement with the investigation in this study. The result is in agreement to the hypothesis that hPNAS-4 gene combined with DDP may exert an enhanced antitumor effect.

Angiogenesis plays a vital role in tumor growth and metastasis because the blood vessels supply malignant cells with sufficient oxygen and nutrients $(27,28)$. Thus, interruption of angiogenesis is an attractive strategy to inhibit tumor growth. In this study, inhibition of angiogenesis by hPNAS-4 was determined by $\mathrm{CD} 31$ and calcium alginate embedding in vivo. Lower microvessel density was manifested in the hPNAS-4 treated group compared with the empty vector, indicating that hPNAS-4 could inhibit angiogenesis. In addition, angiogenesis in the hPNAS-4 plus DDP group was significantly inhibited compared with the other groups. However, the exact anti-angiogenesis mechanism of hPNAS-4 remains unclear.

The mechanism of the combination therapy is not precisely understood. It may be involved multiple pathways. Based on the results of this and previous studies, we hypothesize that: i) overexpression of hPNAS-4 induces tumor cell apoptosis; ii) DDP promotes tumor cell apoptosis; iii) expression of hPNAS-4 is increased when DNA is damaged by DDP, which in turn enhances tumor cell apoptosis; and iv) the efficacy of inhibition of angiogenesis is strengthened by the combination of hPNAS-4 and DDP.

Gene transfer mainly depends on effective gene delivery systems $(14,15)$. Many efforts have been made to utilize non-viral delivery systems, especially biodegradable polymeric nanoparticles (29-31). It has been reported that PLGA nanoparticles have many advantages, such as sustained and gradual release, nontoxicity, biocompatibility, biodegradability, high amounts of plasmid, facilitating cellular uptake and tissue penetrability (17-19,32-36). Additionally, loading PLGA nanoparticles with cDNA could escape from nuclease degradation and maintain the integrity of cDNA conformation. As a delivery system, PLGA has exhibited satisfactory results $(32,37-40)$.

In this study, the physicochemical properties of hPNAS-4 loaded PLGA nanoparticles were assessed by spectroscopy and fluorometric detection. Gene-loaded PLGA nanoparticles in spherical shape with a smooth surface were confirmed by SEM images. Particle size is a crucial parameter of nanoparticles. It can affect drug release, stability during storage, cellular uptake, and biodistribution (41). The size of PLGA nanoparticles around $200 \mathrm{~nm}$ was applicable for biodistribution in vivo. Overexpression of hPNAS-4 mRNA in tumor tissues of mice treated with PLGA nanoparticles encapsulating hPNAS-4 was detected by RT-PCR in vitro and in vivo. The results indicate that PLGA nanoparticles could 
deliver the hPNAS-4 gene into the tumor tissues successfully and the expression of the targeted gene could be detected at the mRNA level. Subsequently, the antitumor efficiency of hPNAS-4 delivered by PLGA was evaluated in a xenograft model of ovarian cancer. The tumor weight in the PhP-P group was lower compared with the P-P group. The tumor growth in the PhP-P group was effectively suppressed by administration of PhP-P. These results suggest that PLGA could successfully transfer the hPNAS-4 gene into tumor cells in vivo.

PLGA, as a co-polymer formed from PLA and PGA, can be degraded into carbon dioxide and water, and excreted through metabolism. It is an efficient gene delivery system without toxicity. In this study, inflammation, or incrassate or hyperemic peritoneum were not observed in any group. There were no significant differences in the body weights of mice among the five groups. Systemic toxicity was not detected. Even though weight loss was observed in the DDP and PhP-P plus DDP-treated groups, it might due to the toxicity of DDP. $\mathrm{PhP}-\mathrm{P}$ showed an excellent tolerance in the course of intraperitoneal treatment. The apparent safety of PLGA suggests it should be tested in future clinical trials.

In conclusion, hPNAS-4 may have an effective antitumor activity and anti-angiogenesis effect against ovarian carcinoma. The antitumor activity of hPNAS-4 was enhanced when combined with DDP. PLGA may exert efficient transfection without gross toxicity. Thus, PLGA delivering hPNAS-4 combined with DDP may have a promising application in the treatment of ovarian cancer.

\section{Acknowledgements}

We thank Dr Zhu Yuan for the expert technical assistance. This study was supported by the National Natural Science Foundation of China (Grant no. NSFC81071861).

\section{References}

1. Yuan Z, Liu H, Yan F, et al: Improved therapeutic efficacy against murine carcinoma by combining honokiol with gene therapy of PNAS-4, a novel pro-apoptotic gene. Cancer Sci 100: 1757-1766, 2009.

2. Yao S, Xie L, Qian M, et al: PNAS-4 is a novel regulator for convergence and extension during vertebrate gastrulation. FEBS Lett 582: 2325-2332, 2008.

3. Yan F, Ruan XZ, Yang HS, et al: Identification, characterization, and effects of Xenopus laevis PNAS-4 gene on embryonic development. J Biomed Biotechnol 2010:134764, 2010.

4. Mo D, Zhu Z, te Pas MF, et al: Characterization, expression profiles, intracellular distribution and association analysis of porcine PNAS-4 gene with production traits. BMC Genet 9: 40, 2008.

5. Yan F, Gou L, Yang J, et al: A novel pro-apoptosis gene PNAS-4 that induces apoptosis in A549 human lung adenocarcinoma cells and inhibits tumor growth in mice. Biochimie 91: 502-507, 2009.

6. Filippov V, Filippova M, Sinha D and Duerksen-Hughes $P$ : PNAS-4: a novel pro-apoptotic gene activated during the early response to DNA damage. Proc Am Assoc Cancer Res 46: 717, 2005.

7. Hou S, Zhao Z, Yan F, Chen X, Deng H, Chen X, Wang Y and Wei Y: Genetic transfer of PNAS-4 induces apoptosis and enhances sensitivity to gemcitabine in lung cancer. Cell Biol Int 33: 276-282, 2009.

8. Jemal A, Siegel R, Xu J and Ward E: Cancer statistics, 2010. CA Cancer J Clin 60: 277-300, 2010.

9. Reed E: Platinum-DNA adduct, nucleotide excision repair and platinum based anti-cancer chemotherapy. Cancer Treat Rev 24 331-344, 1998.
10. Jamieson ER and Lippard SJ: Structure, recognition, and processing of DDP-DNA adducts. Chem Rev 99: 2467-2498, 1999.

11. Brown SJ, Kellett PJ and Lippard SJ: Ixr1, a yeast protein that binds to platinated DNA and confers sensitivity to DDP. Science 261: 603-605, 1993.

12. Ohndorf U-M, Rould MA, He Q, Pabo CO and Lippard SJ: Basis for recognition of DDP-modified DNA by high-mobility-group proteins. Nature 399: 708-712, 1999.

13. Yan F, Qian M, Yang F, et al: A novel pro-apoptosis protein PNAS-4 from Xenopus laevis: cloning, expression, purification, and polyclonal antibody production. Biochemistry (Mosc) 72: 664-671, 2007.

14. Marshall E: Gene therapy. What to do when clear success comes with an unclear risk? Science 298: 510-511, 2002.

15. Ott MG, Schmidt M, Schwarzwaelder K, et al: Correction of X-linked chronic granulomatous disease by gene therapy, augmented by insertional activation of MDS1-EVI1, PRDM16 or SETBP1. Nat Med 12: 401-409, 2006

16. Yang F, Li Z, Deng H, et al: Efficient inhibition of ovarian cancer growth and prolonged survival by transfection with a novel pro-apoptotic gene, hPNAS-4, in a mouse model. Oncology 75: 137-144, 2008

17. Jain RA: The manufacturing techniques of various drug loaded biodegradable poly (lactide-co-glycolide) (PLGA) devices. Biomaterials 21: 2475-2490, 2001.

18. Walter E, Dreher D, Kok M, Thiele L, Kiama SG and Merkle PH: Hydrophilic poly(dl-lactide-co-glycolide) microspheres for the delivery of DNA to human-derived macrophages and dendritic cells. J Control Release 76: 149-168, 2001.

19. Luu YK, Kim K, Hsiao BS, Chu B and Hadjiargyrou M: Development of a nanostructured DNA delivery scaffold via electrospinning of PLGA and PLA-PEG block copolymers. J Control Release 89: 341-353, 2003.

20. Panyam J, Zhou WZ, Prabha S, Sahoo SK and Labhasetwar V: Rapid endolysosomal escape of poly (d,l-lactide-co-glycolide) nanoparticles: implications for drug and gene delivery. FASEB J 16: 1217-1226, 2002

21. Freytag T, Dashevsky A, Tillman L, Hardee GE and Bodmeier R: Improvement of the encapsulation efficiency of oligonucleotidecontaining biodegradable microspheres. J Control Release 69: $197-207,2000$

22. Lin $\mathrm{Xj}$, Chen XC, Wang L, et al: Dynamic progression of an intraperitoneal xenograft model of human ovarian cancer and its potential for preclinical trials. J Exp Clin Cancer Res 26: 467-474, 2007.

23. O'Reilly MS, Boehm T, Shing Y, et al: Endostatin: an endogenous inhibitor of angiogenesis and tumor growth. Cell 88: 277-285, 1997.

24. Hanan SS, Gary JK, Vernon S, Chinthalapally VR and Bandaru SR: Proliferative index in breast carcinoma determined in situ by Ki67 immunostaining and its relationship to clinical and pathological variables. J Pathol 152: 287-295, 1987.

25. Hanan SS, Gary JK, Vernon S, Chinthalapally VR and Bandaru SR: Modulation of apoptosis by sulindac, curcumin, phenylethyl-3-methylcaffeate, and 6-phenylhexyl isothiocyanate: apoptotic index as a biomarker in colon cancer chemoprevention and promotion. Cancer Res 57: 1301-1305, 1997.

26. Yuan Z, Yan F, Wang YS, et al: PNAS-4, a novel pro-apoptotic gene, can potentiate antineoplastic effects of DDP. Cancer Chemother Pharmacol 65: 13-25, 2009.

27. Folkman J: Tumor angiogenesis: therapeutic implications. $\mathrm{N}$ Engl J Med 285: 1182-1186, 1971.

28. Folkman J: What is the evidence that tumors are angiogenesis dependent? J Natl Cancer Inst 82: 4-6, 1990.

29. Langer R: Drug delivery and targeting. Nature 392: 5-10, 1998.

30. Gref R, Minamitake Y, Peracchia MT, Trubetskoy V, Torchilin V and Langer R: Biodegradable long-circulating polymeric nanospheres. Science 263: 1600-1603, 1994.

31. Ferrari M: Cancer nanotechnology: opportunities and challenges. Nat Rev 5: 161-171, 2005.

32. Carrasquillo KG, Ricker JA, Rigas IK, Miller JW and Gragoudas ES, Adamis AP: Controlled delivery of the anti-VEGF aptamer EYE001 with poly(lactic-co-glycolic)acid microspheres. Invest Ophthalmol Vis Sci 44: 290-299, 2003.

33. Davis ME: Non-viral gene delivery systems. Curr Opin Biotechnol 13: 128-131, 2002.

34. Schmidt-Wolf GD and Schmidt-Wolf IG: Non-viral and hybrid vectors in human gene therapy: an update. Trends Mol Med 9: 67-72, 2003. 
35. Basarkar A and Singh J: Nanoparticulate systems for polynucleotide delivery. Int J Nanomedicine 2: 353-360, 2007.

36. Shive MS and Anderson JM: Biodegradation and biocompatibility of PLA and PLGA microspheres. Adv Drug Deliv Rev 28: 5-24, 1997.

37. Choleris E, Little RS, Mong JA, Puram SV, Langer R and Pfaff DW. Microparticle-based delivery of oxytocin receptor antisense DNA in the medial amygdala blocks social recognition in female mice. Proc Natl Acad Sci USA 11: 4670-4675, 2007.

38. Liu J, Meisner D, Kwong E, Wu XY and Johnston MR: Translymphatic chemotherapy by intrapleural placement of gelatin sponge containing biodegradable paclitaxel colloids controls lymphatic metastasis in lung cancer. Cancer Res 69: 1174-1181, 2009.
39. Amaral AC, Bocca AL, Ribeiro AM, et al: Amphotericin B in poly (lactic-co-glycolic acid) (PLGA) and dimercaptosuccinic acid (DMSA) nanoparticles against paracoccidioidomycosis. J Antimicrob Chemother 63: 526-533, 2009.

40. Du L, Cheng J, Chi Q, Qie J, Liu Y and Mei X: Biodegradable PLGA microspheres as a sustained release system for a new luteinizing hormone-releasing hormone (LHRH) antagonist. Chem Pharm Bull 54: 1259-1265, 2006.

41. Feng SS: Nanoparticles of biodegradable polymers for new concept chemotherapy. Expert Rev Med Devices 1: 115-125, 2004. 